\title{
Clinical and evoked potential changes in acute transverse myelitis following methyl prednisolone
}

\author{
J Kalita ${ }^{1}$, PM Guptar ${ }^{1}$ and UK Misra*,1 \\ ${ }^{1}$ Department of Neurology, Sanjay Gandhi PGI of Medical Sciences, India
}

\begin{abstract}
Study design: Observational study with sequential follow-up.
Objective: To study the role of somatosensory evoked potential (SEP) and motor evoked potential (MEP) in monitoring the effect of methyl prednisolone (MPS) therapy in acute transverse myelitis (ATM).

Setting: Tertiary care referral teaching hospital at Lucknow, India.

Methods: In the present study, nine patients with ATM whose age ranged between 12 and 42 years and three of whom were females have been included. They were subjected to clinical examination, median and tibial SEP and Central motor conduction time (CMCT) to upper and lower limbs. The clinical and evoked potential studies were repeated after 7 and 90 days of intravenous methyl prednisolone treatment for 5 days. The outcome was defined on the basis of a 3 month Barthel Index $(\mathrm{BI})$ score into poor $(\mathrm{BI}<12)$ and $\operatorname{good}(\mathrm{BI} \geqslant 12)$.

Results: All the patients had varying degrees of leg weakness ranging between grade 0 and 4 on the Medical Research Council scale. Upper limbs were weak in four patients. Pinprick and joint position sensations in the lower limb were impaired in all patients. Central motor conduction time to upper limb was abnormal in two patients and to lower limbs in eight patients. Median SEPs were normal in all and tibial in two patients. On the seventh day follow-up, muscle power improved in six which correlated with CMCT-to tibialis anterior in five patients. Joint position sense improved in two patients on the seventh day but there was no further improvement at 3 months. Tibial SEP, however, improved in four patients on the seventh day and six patients at 3 months. Following methyl prednisolone therapy, both sensory and motor functions improved, but the improvement was more pronounced and more frequent at 3 months compared to that on seventh day. At a 3 month follow-up, six patients had good and three poor recovery.
\end{abstract}

Conclusion: Evoked potential studies provide additional objective means for monitoring the effect of therapy in ATM.

Keywords: transverse myelitis; corticosteroid; evoked potentials; prognosis

\section{Introduction}

Acute transverse myelitis (ATM) is an acute or subacute spinal cord dysfunction characterised by paraplegia, horizontal level of sensory impairment and sphincter disturbance in which secondary causes such as compressive lesions, tuberculosis, syphilis arteriovenous malformation, trauma and malignant infiltration have been excluded. ${ }^{1}$ The sensory functions can be objectively documented by somatosensory evoked potential (SEP) and motor functions by motor evoked potential (MEP) studies. Median SEPs have been reported to be normal in ATM. However, tibial SEPs are frequently abnormal., ${ }^{2,3}$ Motor evoked potentials are more frequently abnormal and, if unrecordable especially on spinal stimulation, predict a poor prognosis. Corticosteroids are frequently used in the management of ATM. ${ }^{2,4,5}$ The objective

*Correspondence: UK Misra, Department of Neurology, Sanjay Gandhi PGI of Medical Sciences, Lucknow - 226 014, India documentation of sensory and motor functions employing evoked potential studies in patients with ATM may be useful. In this communication we report sequential clinical, SEP and MEP findings in patients with ATM following a short course of methyl prednisolone (MPS) therapy.

\section{Patients and methods}

Nine patients with ATM within 14 days of the onset of illness have been included in this study. The diagnosis of ATM was based on the criteria of Jeffery et al. ${ }^{6}$ All the patients underwent a detailed neurological examination. Weakness was graded as per 0-5 Medical Research Council (MRC) scale and tone by Ashworth scale. $^{7}$ Tendon reflex, pinprick, sense of joint position and vibration were recorded. Blood counts, serum electrolyte, VDRL, collagen profile and ELISA for HIV was carried out in all the patients. Cerebrospinal 
fluid was examined for cell, protein, sugar and culture for bacteria and fungi. Spinal MRI was carried out on a $2.0 \mathrm{~T}$ superconducting system operating at $1.5 \mathrm{~T}$ (Magnetome, SP, Siemens, Germany) using a flat oval surface coil. All images were obtained employing multislice spin echo sequences (SE) which included gradient motion rephasing to reduce motion induced artefact. T1 (500/15/3, TR in $\mathrm{ms} / \mathrm{TE}$ in $\mathrm{ms} /$ excitation $)$, proton density $(2200-2500 / 15-20 / 1)$ and $\mathrm{T} 2$ weighted (2200-2500/80-90/1) SE images were obtained in the sagittal plane with slice thickness of $3 \mathrm{~mm}$ interslice gap of $0.3 \mathrm{~mm}$ and $220 / 256 \times 256$ matrix. The whole spinal cord imaging was completed in two to three examinations.

\section{Median and tibial somatosensory evoked potential} Median SEPs were obtained by stimulating the median nerve at the wrist by a $0.1 \mathrm{~ms}$ square wave pulse at $3 \mathrm{~Hz}$ at an intensity to produce a painless twitch of the thumb. The active surface recording electrode was placed at Erb's point and at contralateral parietal cortex $3 \mathrm{~cm}$ behind and $7 \mathrm{~cm}$ lateral to vertex using a midfrontal reference. For tibial SEPs, the posterior tibial nerve was stimulated below the medial malleolus at $3 \mathrm{~Hz}$ sufficient to produce a painless twitch of the big toe. The recording electrode was placed on the spinous process of the first lumbar vertebra $\left(\mathrm{L}_{1}\right)$ and $2 \mathrm{~cm}$ caudal to $\mathrm{Cz}\left(\mathrm{Cz}^{\prime}\right)$. The reference electrodes were placed at $\mathrm{L}_{3}$ and $\mathrm{Fz}$ respectively. The impedence of the electrode was kept below $5 \mathrm{~K} \Omega$, the frequency bandpass was $2-3000 \mathrm{~Hz}$ and analysis time $100 \mathrm{~ms}$. Five hundred and twelve responses were twice averaged at a gain of $1-2 \mu \mathrm{V} /$ division to ensure reproducibility employing Neuromatic 2000 (Dantec, Denmark). Median SEPs were analyzed by the latency of $\mathrm{N}_{9}, \mathrm{~N}_{20}$ and interpeak latency of $\mathrm{N}_{9}-\mathrm{N}_{20}$. For tibial SEPs latencies $\mathrm{N}_{21}, \mathrm{P}_{40}$ and $\mathrm{N}_{21}-\mathrm{P}_{40}$ conduction time (Central sensory conduction time) were measured. ${ }^{3}$

\section{Motor evoked potential (MEP)}

Motor evoked potentials were recorded from both upper and lower limbs bilaterally following transcranial electrical stimulation of the cortex and spine. A Digitimer D-180 (England) stimulator delivering an electrical shock up to $750 \mathrm{~V}$ with a time constant of $50-100 \mu$ s was used. The stimulating electrode was a $1 \mathrm{~cm}$ diameter saline soaked with felt pads mounted on a plastic handle. To activate abductor digiti minimi (ADM), the cathode was placed at the vertex and anode $7 \mathrm{~cm}$ laterally and $1 \mathrm{~cm}$ anterior to a line drawn from the vertex to tragus. For activating tibialis anterior (TA) the anode was kept at the vertex and cathode $7 \mathrm{~cm}$ posterior. For cervical and lumbar stimulation the cathode was placed below the spinous process of the seventh cervical $\left(\mathrm{C}_{7}\right)$ and twelfth thoracic vertebra $\left(\mathrm{T}_{12}\right)$ respectively and the anode proximal. Motor evoked potentials were recorded by surface electrodes, placed on ADM or TA in a belly tendon montage. During the cortical stimulation, the patient was asked to contract the target muscle slightly $(10 \%$ of the maximum force irrespective of degree of weakness). During spinal stimulation, the patient was asked to relax. Electromyogram signals were filtered through $20 \mathrm{~Hz}-2 \mathrm{KHz}$ at a gain of $0.5-1 \mathrm{mV} /$ division. The stimulus intensity was $90-100 \%$ for cortical and $50-60 \%$ of the maximum output for spinal stimulation. Three responses were obtained at $10 \mathrm{~s}$ intervals and the one with the shortest latency was recorded. Onset latency and the amplitude of the negative phase was measured. Central motor conduction time to abductor digiti minimi (CMCT-ADM) was calculated by subtracting the latency on cervical $\left(\mathrm{C}_{7}\right)$ stimulation from that on cortical stimulation and that for the Tibialis anterior (CMCT-TA) by subtracting the latency on lumbar $\left(\mathrm{L}_{1}\right)$ stimulation from that on vertex stimulation. ${ }^{3}$

The reports of evoked potentials were compared with the normal values of our laboratory which were obtained from 32 healthy adult volunteers. Their age ranged between 15 and 60 years. The upper limit of normal was defined by mean $\pm 2.5 \mathrm{SD}$ of controls. The upper limit of CMCT-ADM (mean \pm SD) was 8.1 $(5.1 \pm 1.2) \mathrm{ms}$, CMCT-TA $16.1(12.1 \pm 1.6) \mathrm{ms}$, median N9-N20 conduction time $11.3(8.3 \pm 1.2) \mathrm{ms}$ and tibial N21-P40 conduction time was $27.1(20.1 \pm 2.8) \mathrm{ms}$.

In the follow-up studies, the improvement in evoked potentials was defined if the change exceeded the normal intra-individual variation. To determine the normal intra-individual variation, normal volunteers were subjected to MEP and SEP studies for three consecutive days and the maximum difference was analysed. The mean (SE) of maximum difference for CMCT-TA was 0.79 (0.15) $\mathrm{ms}$ and that for tibial central sensory conduction time (CSCT) was 0.53 (0.14) ms. The upper limit of normal intra-individual variability was defined as mean $+2.576 \mathrm{SE}$ which covers $99 \%$ of normal variability. ${ }^{8}$ The relationship between evoked potential with clinical variables was evaluated by chi square $(\chi 2)$ test. The extent of MRI changes (five spinal segments or more) were correlated with 3 month outcome employing $\chi 2$ test.

\section{Treatment and follow-up}

All the patients received methylprednisolone $500 \mathrm{mg}$ intravenously injected over $6 \mathrm{~h}$ for five consecutive days. The clinical and evoked potential studies were carried out before MPS therapy and repeated on 7 and 90 days after the treatment.

\section{Outcome}

The outcome was defined on the basis of a 3 month Barthel Index score. A score below 12 indicated poor and 12 or more as good recovery. ${ }^{3}$ 


\section{Results}

Nine patients with ATM received MPS therapy of whom three were females. Their age ranged between 12 and 42 (mean 25.5) years. History of fever was present in five patients and pain and paresthesiae in seven. The weaknesses peaked by 1-14 (mean 3.9) days. All the patients had varying degrees of weakness ranging between grade 0 and 4 . Upper limbs were weak in four patients; one of whom had pronounced weakness with respiratory paralysis and the remaining had mild weaknesses (grade 4). Muscle tone in lower limbs was reduced in six, normal in two and increased in one patient. Lower limb tendon reflexes were reduced in four and exaggerated in three patients. Upper limb reflexes were normal in all except two patients who had hyperreflexia. Horizontal level of sensory loss was present in all the patients and ranged between first lumbar and sixth cervical spinal level. Joint position sensations in lower limbs were impaired and there was urinary retention in all the patients. Cerebrospinal fluid was abnormal in eight patients. The mean cell count was $28(5-250) / \mathrm{mm}^{3}$, protein 120 (range $\left.20-300\right) \mathrm{mg} / \mathrm{dl}$ and sugar 50 (range $40-60) \mathrm{mg} / \mathrm{dl}$. Cerebrospinal fluid was negative for bacterial and fungal cultures, HIV and VDRL in all the patients. Collagen profile was also negative in all the patients. Spinal MRI was carried out in eight patients. On T2 sequence there were hyperintense signal changes extending from 2-18 spinal segments. The signal changes were present in the cervical region in five, dorsal in five and lumber in one patient.

\section{Evoked potential changes}

Motor evoked potentials were more frequently abnormal compared to SEP. The MEP was abnormal in eight patients whereas SEP in six. The upper limb central motor conduction time was abnormal in two patients; prolonged bilaterally in one and unilaterally in the other. Lower limb CMCT was abnormal in eight patients, unrecordable in five patients (nine sides) and prolonged in three patients (six sides). Median SEPs were normal in all and tibial were abnormal in six patients; unrecordable in five (eight sides) and prolonged in one patient (one side).

\section{Effect of methyl prednisolone therapy}

Following MPS therapy, there was I MRC grade improvement of lower limb power in six patients (12 sides), which correlated with improvement in CMCTTA in four patients (six sides). Both the patients with upper limb CMCT abnormalities improved following MPS treatment with respect to muscle power and CMCT-ADM values. In two patients, clinical improvement was not associated with MEP improvement. On the other hand, in 1 patient whose MEP improved, there was no clinical recovery. At 3 months, further improvement was noted in muscle power in eight patients which ranged between grade 1 and 5. It correlated with CMCT improvement in five patients (nine sides).

Following MPS therapy joint position sensation improved in two patients. Tibial SEP, however, improved in four patients (4 sides) becoming recordable in one and in the remaining there was improvement in CSCT. At the 3 months follow-up, tibial CSCT improved further in six patients (ten sides). CMCT and CSCT although improved on seventh day after MPS therapy, however, the improvement was more marked at the 3 month follow-up. The sequential changes in lower limb

Table 1 Sequential changes in CMCT-TA and lower limb power in patients with acute transverse myelitis

\begin{tabular}{|c|c|c|c|c|c|c|c|c|}
\hline $\begin{array}{l}\text { Sl. } \\
\text { No. }\end{array}$ & $0 d a y$ & $\begin{array}{c}C M C T-T A \\
7 \text { davs }\end{array}$ & 90 davs & 0 dav & $\begin{array}{c}\text { uscle Po } \\
7 \text { days }\end{array}$ & 90 davs & $\begin{array}{l}\text { Extent of } \\
\text { MRI change }\end{array}$ & $\begin{array}{c}\text { Outcome } \\
(B I)\end{array}$ \\
\hline \multirow[t]{2}{*}{1} & R 14.0 & 12.8 & 12.8 & 4 & 5 & 5 & $\mathrm{C}_{2}-\mathrm{C}_{6}$ & \\
\hline & L 13.6 & 16.0 & 12.6 & 4 & 5 & 5 & & good (20) \\
\hline \multirow[t]{2}{*}{2} & R NR & NR & NR & 0 & 1 & 2 & $\mathrm{D}_{4}-$ conus & \\
\hline & L NR & NR & 13.2 & 0 & 1 & 2 & & poor (4) \\
\hline \multirow[t]{2}{*}{3} & R 36.4 & 32.8 & 24.8 & 3 & 4 & 5 & $\mathrm{C}_{1}-\mathrm{C}_{8}$ & \\
\hline & L 36.0 & 32.8 & 30.0 & 3 & 4 & 5 & & good $(20)$ \\
\hline \multirow[t]{2}{*}{4} & R NR & NR & 14.0 & 0 & 0 & 5 & $C_{1}-D_{1}$ & \\
\hline & L NR & NR & 17.2 & 0 & 0 & 5 & & good $(20)$ \\
\hline \multirow[t]{2}{*}{5} & R NR & 13.2 & 13.6 & 0 & 1 & 2 & $\mathrm{D}_{2}-\mathrm{D}_{9}$ & \\
\hline & L NR & NR & NR & 0 & 1 & 2 & & poor (4) \\
\hline \multirow[t]{2}{*}{6} & R 14.4 & 13.6 & 12.8 & 2 & 2 & 4 & $\mathrm{D}_{5}-\mathrm{D}_{6}$ & \\
\hline & L NR & 14.4 & 14.8 & 2 & 2 & 4 & & good (19) \\
\hline \multirow[t]{2}{*}{7} & R NR & NR & NR & 0 & 1 & 3 & ND & \\
\hline & L NR & NR & NR & 0 & 1 & 3 & & poor (4) \\
\hline \multirow[t]{2}{*}{8} & R 18.0 & 20.8 & 12.4 & 4 & 4 & 5 & $\mathrm{C}_{2}-\mathrm{C}_{8}$ & \\
\hline & L 16.4 & 20.0 & 17.6 & 4 & 4 & 5 & & good (20) \\
\hline \multirow[t]{2}{*}{9} & R 32.0 & 24.4 & 9.6 & 2 & 3 & 5 & $C_{3}-D_{12}$ & \\
\hline & L 24.0 & 13.0 & 8.8 & 2 & 3 & 5 & & good (20) \\
\hline
\end{tabular}

$\mathrm{NR}=$ not recordable, $\mathrm{BI}=$ Barthel index score at 3 months, $\mathrm{ND}=$ not done, $\mathrm{R}=$ right, $\mathrm{L}=$ left 
muscle power, CMCT-TA, joint position sensations and tibial CSCT are shown in Tables 1 and 2. At the 3 months follow-up six patients had good and three poor recovery.

In two patients, the MRI changes were restricted to five spinal segments or less and both recovered well by 3 months. Five out of six patients with extensive signal alternation on spinal MRI exceeding five segments also recovered well. The extent of MRI signal changes did not correlate with outcome $\left(\chi^{2}=0.38\right.$, df-1, NS). MEP correlated with the degree of weakness $\left(\chi^{2}=9.0\right.$, $\mathrm{df}=2, \quad P<0.01)$ and the outcome $\left(\chi^{2}=9.0, \mathrm{df}=2\right.$, $P<0.01)$; whereas SEP neither correlated with sensory loss $\left(\chi^{2}=2, \mathrm{df}=2, \mathrm{NS}\right)$ nor with outcome $\left(\chi^{2}=1.3\right.$, $\mathrm{df}=2, \mathrm{NS})$.

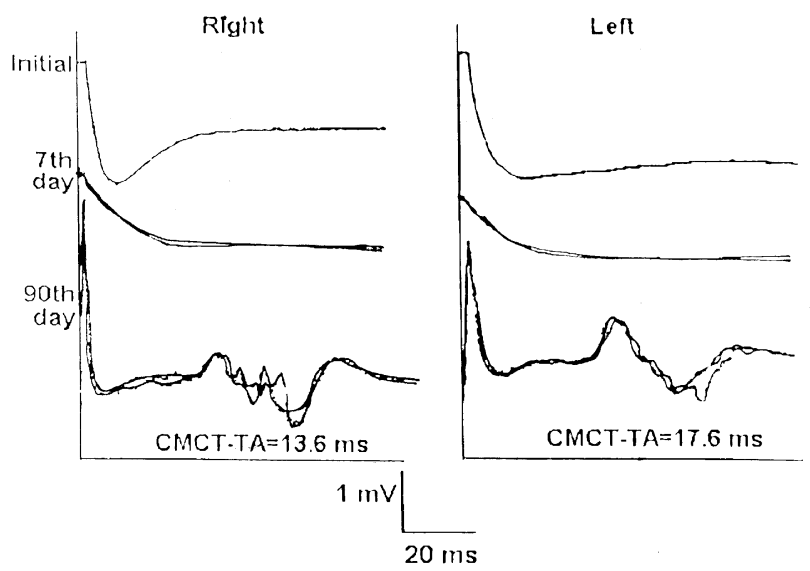

Figure 1 Effect of methyl prednisolone therapy in acute transverse myelitis (patient no. 4). Unrecordable CMCT-TA became recordable at a 3 month follow-up but was prolonged in the left side. The patient recovered completely at 3 months

\section{Discussion}

In our study, the lower limb MEP and SEP were more frequently abnormal compared to the upper limb. This could be due to more severe involvement of the thoracid region of the spinal cord in ATM. The involvement of the cervical region of the spinal cord in ATM has been reported to be common if MRI and evoked potential changes are also included. ${ }^{3}$ Of the two evoked potentials, MEPs were more frequently abnormal (eight out of nine patients) compared to the SEP (six out of nine patients). Two main abnormalities in evoked potentials were noted; inexcitable sensory or motor pathways and prolonged central conduction time. Prolongation of conduction time may be due to demyelination, damage to fast conducting pathways and desynchronisation of descending volleys. ${ }^{9}$ Unrecordable evoked potentials may be due to damage in fast conducting pathways. In ATM, a varying degree of white and gray matter changes in the spinal cord have been reported. ${ }^{10}$ Presence of white matter changes may lead to prolongation of CMCT or CSCT whereas combined white and gray matter involvement may lead to unrecordable SEP, MEP or both. ${ }^{3}$

On the seventh day of intravenous MPS therapy, six patients improved clinically. Motor evoked potential improvement, however, was noted in four of these patients. In two patients, clinical improvement was not associated with MEP improvement. On the other hand, in one patient, MEP improved but there was no clinical recovery. In CMCT studies, the fastest conducting fibres are evaluated which are responsible for phasic movements; whereas muscle power testing relies on tonic muscle contraction which is a function of slow conducting motor pathways. ${ }^{10,11}$ This can

Table 2 Sequential changes in tibial CSCT and lower limb joint position sense in the patients with acute transverse myelitis

\begin{tabular}{|c|c|c|c|c|c|c|c|c|}
\hline \multirow{2}{*}{$\begin{array}{l}\text { Sl. } \\
\text { No. }\end{array}$} & \multicolumn{3}{|c|}{ Tibial CSCT } & \multicolumn{3}{|c|}{ Joint position sense } & \multirow{2}{*}{$\begin{array}{l}\text { Extent of } \\
\text { MRI change }\end{array}$} & \multirow{2}{*}{$\begin{array}{l}\text { Outcome } \\
\text { (BI) }\end{array}$} \\
\hline & 0 day & 7 days & 90 days & 0 day & 7 days & 90 days & & \\
\hline \multirow[t]{2}{*}{1} & R 24.4 & 24.4 & 20.0 & $a b$ & $a b$ & $a b$ & \multirow[t]{2}{*}{$\mathrm{C}_{2}-\mathrm{C}_{6}$} & \\
\hline & L 23.2 & 21.0 & 20.4 & $a b$ & $\mathrm{ab}$ & $\mathrm{ab}$ & & $\operatorname{good}(20)$ \\
\hline \multirow[t]{2}{*}{2} & R NR & NR & NR & $a b$ & $a b$ & $a b$ & \multirow[t]{2}{*}{$\mathrm{D}_{4}-$ conus } & \\
\hline & L NR & NR & NR & $a b$ & $\mathrm{ab}$ & $\mathrm{ab}$ & & poor (4) \\
\hline \multirow[t]{2}{*}{3} & R NR & NR & 35.0 & $a b$ & $a b$ & $a b$ & \multirow[t]{2}{*}{$\mathrm{C}_{1}-\mathrm{C}_{8}$} & \\
\hline & L NR & NR & 24.4 & $\mathrm{ab}$ & $a b$ & $\mathrm{ab}$ & & $\operatorname{good}(20)$ \\
\hline \multirow[t]{2}{*}{4} & R 24.0 & 23.8 & 19.6 & $a b$ & $a b$ & $\mathrm{ab}$ & \multirow[t]{2}{*}{$\mathrm{C}_{1}-\mathrm{D}_{1}$} & \\
\hline & L 31.2 & 24.4 & 19.8 & $a b$ & $a b$ & $\mathrm{ab}$ & & $\operatorname{good}(20)$ \\
\hline \multirow[t]{2}{*}{5} & R 17.6 & 17.6 & 15.6 & $a b$ & $\mathrm{~N}$ & $\mathrm{~N}$ & \multirow[t]{2}{*}{$\mathrm{D}_{2}-\mathrm{D}_{9}$} & \\
\hline & L 18.2 & 18.8 & 15.6 & $a b$ & $\mathrm{~N}$ & $\mathrm{~N}$ & & poor (4) \\
\hline \multirow[t]{2}{*}{6} & R NR & NR & NR & $a b$ & $a b$ & $\mathrm{ab}$ & \multirow[t]{2}{*}{$\mathrm{D}_{5}-\mathrm{D}_{6}$} & \\
\hline & L NR & NR & NR & $a b$ & $a b$ & $a b$ & & $\operatorname{good}(19)$ \\
\hline \multirow[t]{2}{*}{7} & R NR & NR & NR & $a b$ & $a b$ & $\mathrm{ab}$ & \multirow[t]{2}{*}{ ND } & \\
\hline & L NR & NR & NR & $a b$ & $a b$ & $a b$ & & poor (4) \\
\hline \multirow[t]{2}{*}{8} & R NR & 26.6 & 21.2 & $a b$ & $\mathrm{~N}$ & $\mathrm{~N}$ & \multirow[t]{2}{*}{$\mathrm{C}_{2}-\mathrm{C}_{8}$} & \\
\hline & L 19.2 & 22.4 & 22.4 & $a b$ & $\mathrm{~N}$ & $\mathrm{~N}$ & & $\operatorname{good}(20)$ \\
\hline \multirow[t]{2}{*}{9} & R 21.4 & 20.4 & 18.8 & $a b$ & $a b$ & $a b$ & \multirow[t]{2}{*}{$C_{3}-D_{12}$} & \\
\hline & L 22.0 & 21.6 & 18.8 & $a b$ & $a b$ & $a b$ & & $\operatorname{good}(20)$ \\
\hline
\end{tabular}

$\mathrm{NR}=$ not recordable, $\mathrm{ab}=$ abnormal, $\mathrm{N}=$ normal, $\mathrm{BI}=$ Barthel index score at 3 months, $\mathrm{ND}=$ not done, $\mathrm{R}=\mathrm{right}, \mathrm{L}=$ left 
account for the occasional discrepancy between muscle power and CMCT. Clinical sensory testing was unchanged at 1 week; however, CSCT improved in four patients. The early improvement after MPS therapy may be due to antioedema effect and clearing of conduction block.

At the 3 months follow-up, further improvement was noted in both MEP and SEP. The MEP improvement was associated with clinical improvement in five patients. The improvement at 3 months was more pronounced compared to that at 7 days after MPS therapy. The improvement at this stage may be multifactorial and may depend on remyelination, collateral sprouting and neuronal plasticity. The role of MPS in multiple sclerosis is well documented and its beneficial role in ATM has also been reported. ${ }^{4,5,12,13}$ More pronounced clinical improvement at 3 months compared to 7 days after MPS therapy may be due to a natural process of improvement although a delayed beneficial effect of MPS cannot be ruled out. The answer to this question requires a double blind controlled trial. The natural history of ATM is variable ranging between complete recovery to disabling neurological deficit; About one third of patients with ATM have been reported to have a good recovery, one third fair and one third poor or no recovery. $^{14}$

In the present study, only three patients had a poor recovery. In an earlier study on ATM in which corticosteroids were not used, seven out of ten patients had a poor outcome suggesting a possible role of MPS in ATM. In the earlier study, CMCT-TA improved in two patients and CSCT only in one side at a 3 month follow-up. ${ }^{3}$ The present study suggests a possible beneficial effect of Methyl prednisolone in ATM. A larger controlled study, however, is needed to evaluate further the role of MPS in ATM.

\section{References}

1 Dawson DM, Potts FM. Acute nontraumatic myelopathies. In: Woolsey RM and Young RR (eds). Neurology Clinics. Disorders of spinal cord. WB Saunders, Philadelphia 1991; pp 551-603.

2 Ropper AH, Miett T, Chiappa KH. Absence of evoked potential abnormalities in acute transverse myelopathy. Neurology (Minneap) 1982; 32: $80-82$.

3 Misra UK, Kalita J, Kumar S. A clinical, MRI and neurophysiological study of acute transverse myelitis. J Neurol Sci 1996; 138: $150-156$.

4 Dowling PC, Busch VV, Cook SD. Possible beneficial effect of high dose intravenous steroid therapy in acute demyelinating disease and transverse myelitis. Neurology 1980; 30: 33-36.

5 Choi KH et al. Idiopathic transverse myelitis. MR characteristics. AJNR 1996; 17: $1151-1160$.

6 Jeffery DR. Mandler RN, Davis L. Transverse myelitis retrospective analysis of 33 cases with differentiation of cases associated with multiple sclerosis and parainfectious events. Arch Neurol 1993; 50: $532-535$.

7 Penn RD et al. Intrathecal baclofen for severe spinal spasticity. New Engl J Med 1989; 320: 1517 - 1521.

8 Misra UK, Kalita J. Motor evoked potential is useful for monitoring the effect of collar therapy in cervical spondylotic myelopathy. J Neurol Sci 1998; 154: 222-228.

9 Thompson PD et al. Examination of motor functions in lesions of spinal cord by the stimulation of the motor cortex. Ann Neurol 1987; 21: 389-396.

10 Hoffman HL. Acute necrotic myelopathy. Brain 1955; 78: $377-$ 393.

11 Bantivolgia $\mathrm{M}$. The anatomical organisation of corticospinal connections. In: Rossini PM and Marsden CD (eds). Non invasive stimulation of brain and spinal cord. Fundamentals and clinical applications. Allen R Liss, New York 1998: pp 1-22.

12 Milligan NM, Newcombe R, Compron DA. A double blind control trial of high dose methyl prednisolone in patients with multiple sclerosis I. Clinical effects. J Neurol Neurosurg Psychiat 1987; 50: $511-516$.

13 Barnes MP et al. Intravenous methyl prednisolone for multiple sclerosis in relapse. J Neurol Neurosurg Psychiat 1985; 48: 157 159.

14 Altrocchi PH, Calif PA. Acute transverse myelopathy. Arch Neurol 1963; 9: 111-119. 